4th International Conference Photoinduced Phase Transitions and Cooperative Phenomena, Wrocław 2011

\title{
Interplay between Correlated Electrons and Quantum Phonons in Charge-Ordered and Mott-Insulating Organic Compounds
}

\author{
K. Yonemitsu ${ }^{a, b, c, *}$, N. Maeshima ${ }^{d, e}$ And Y. TAnAKA ${ }^{a, b, c}$ \\ ${ }^{a}$ Institute for Molecular Science, Okazaki 444-8585, Japan \\ ${ }^{b}$ Graduate University for Advanced Studies, Okazaki 444-8585, Japan \\ ${ }^{c}$ JST, CREST, Tokyo 102-0076, Japan \\ ${ }^{d}$ Institute of Materials Science, University of Tsukuba, Tsukuba 305-8573, Japan \\ ${ }^{e}$ Center for Computational Sciences, University of Tsukuba, Tsukuba 305-8577, Japan
}

\begin{abstract}
At an early stage of the photoinduced transition from an insulator to a metal in quasi-two-dimensional organic conductors, a coherent motion of electrons is observed in a charge-ordered insulator, but not so far in a Mott insulator. The mechanisms of these different photoinduced charge dynamics are theoretically studied by numerical solutions to the time-dependent Schrödinger equation for exact many-electron-phonon wave functions on small clusters of model systems. We use two-dimensional three-quarter-filled extended Holstein-Hubbard models on anisotropic triangular lattices. For a charge-ordered insulator on a lattice simplified from the structure of $\alpha$-(BEDT$-\mathrm{TTF})_{2} \mathrm{I}_{3}$, we indeed find a low-energy collective electronic motion coupled with quantum phonons even if the energy of photoexcitation is away from this energy. For a Mott insulator on a lattice simplified from the structure of $\kappa$-(BEDT-TTF $)_{2} \mathrm{X}$, however, such a collective motion does not appear, and quantum phonons are hardly excited.
\end{abstract}

PACS: 78.20.Bh, 71.30.+h, 71.45.Lr, 71.10.Fd

\section{Introduction}

In order to manipulate electronic phases efficiently on the femtosecond time scale, we need to know possible coherence in the motion of many electrons and/or quantum phonons. Most of the experimental and theoretical studies on photoinduced phase transitions [1], except for few works [2], have not paid much attention to the electronic coherence because it is generally believed to decay so quickly that it is quite difficult to observe. However, quite recently, a motion of correlated electrons is observed by pump-probe spectroscopy using few-optical-cycle infrared pulses for a charge-ordered-insulator phase of an organic compound, $\alpha$-(BEDT-TTF $)_{2} \mathrm{I}_{3}$ [BEDT$-\mathrm{TTF}=$ bis(ethylenedithio)-tetrathiafulvalene] [3]. Such a motion has not so far been observed for a Mott-insulator phase of any material. In this context, the understanding of the mechanisms of these different photoinduced charge dynamics is currently and highly desirable.

The organic BEDT-TTF compounds are good targets for comparative studies because they have rich phase diagrams ranging from charge-ordered [4] and the Mott [5] insulators, metals, and superconductors. Electron transfers between molecules and high-frequency molecular vibrations are energetically comparable and interfere with each other in $\alpha$-(BEDT-TTF $)_{2} \mathrm{I}_{3}[3]$. The BEDT-

* corresponding author; e-mail: kxy@ims.ac.jp
-TTF molecule is a constituent molecule for $\kappa$-(BEDT-TTF $)_{2} \mathrm{X}$, which shows a Mott-insulator phase and a photoinduced transition from it to a metallic phase [6]. They have basically equal strengths of couplings between an electron in the highest-occupied molecular orbital and the highest frequency molecular vibration. Then, the degree of quantum interference between electrons and phonons can be compared directly for different electronic phases. Here, we theoretically compare the early-stage charge and phonon dynamics in these phases, bearing the BEDT-TTF compounds in mind.

\section{Model and method}

We use two-dimensional three-quarter-filled extended Holstein-Hubbard models on anisotropic triangular lattices in Refs. $[7,8]$ for $\alpha$-(BEDT-TTF $)_{2} \mathrm{I}_{3}$ and in Ref. [9] for $\kappa$-(BEDT-TTF $)_{2} \mathrm{X}$ :

$$
\begin{aligned}
H & =\sum_{\langle i j\rangle \sigma} t_{i j}\left(c_{i \sigma}^{+} c_{j \sigma}+c_{j \sigma}^{+} c_{i \sigma}\right)+U \sum_{i} n_{i \uparrow} n_{i \downarrow} \\
& +\sum_{\langle i j\rangle} V_{i j} n_{i} n_{j}+g \sum_{i}\left(b_{i}+b_{i}^{+}\right)\left(n_{i}-3 / 2\right) \\
& +\omega_{b} \sum_{i} b_{i}^{+} b_{i},
\end{aligned}
$$

where $c_{i \sigma}^{+}$creates an electron with spin $\sigma$ at site $i$, $n_{i \sigma}=c_{i \sigma}^{+} c_{i \sigma}$, and $n_{i}=\sum_{\sigma} n_{i \sigma}$. The parameter $t_{i j}$ denotes the transfer integral, $U$ - the on-site Coulomb repulsion, and $V_{i j}$ — the intersite Coulomb repulsion, 
which is $V_{p}\left(V_{c}\right)$ for the diagonal (vertical) nearest neighbors in $\alpha$-(BEDT-TTF $)_{2} \mathrm{I}_{3}$ and $V_{b 1}\left(V_{b 2}, V_{p}, V_{q}\right)$ for the intradimer (interdimer) nearest neighbors in $\kappa$-(BEDT-TTF $)_{2} \mathrm{X}$. In the latter, we assume $V_{i j}$ to be $V_{i j}=$ $V_{0} /\left|\boldsymbol{r}_{i}-\boldsymbol{r}_{j}\right|$ with intermolecular distances taken from Ref. [10]. The operator $b_{i}^{+}$creates a phonon (molecular vibration) at site $i$. The parameter $g$ and $\omega_{b}$ denote the electron-phonon coupling strength and the bare phonon energy, respectively. Here we restrict the number of phonons up to three and assume that electrons in two equivalent molecules in the eight-site periodic cluster are coupled with the same phonon mode. This approximation gives quantitatively good results for equilibrium quantities and response functions, and qualitatively acceptable results for photoinduced dynamics. Let us note that the couplings with low-energy classical lattice phonons in Refs. $[7,8]$ are ignored in the present model.

For the model parameters, we use $t_{b 1}=0.06, t_{b 1^{\prime}}=$ $0.08, t_{b 2}=0.16, t_{b 2^{\prime}}=0.18$, and otherwise $t_{i j}=0$, in the notations of Refs. [7, 8] and $t_{b 1}=-0.28, t_{b 2}=-0.10$, $t_{p}=-0.11$, and otherwise $t_{i j}=0$, in the notations of Ref. [9]. Hereafter, we take $\mathrm{eV}$ as the unit of energy. The other parameters are $U=0.7, V_{p}=0.3, V_{c}=0.35$, $V_{b 1}=0.42$, and $\omega_{b}=0.2$.

The time-dependent Schrödinger equation is numerically solved for the many-electron-phonon wave function on the cluster of eight sites with periodic boundary condition, by expanding the exponential evolution operator with time slice $\mathrm{d} t=0.02 \mathrm{eV}^{-1}$ to the 15 th order and by checking the conservation of the norm. Photoexcitation is introduced through the Peierls phase with the time-dependent vector potential,

$$
\begin{aligned}
& \boldsymbol{A}(t)=\left(\boldsymbol{F} / \sqrt{2 \pi} \omega_{\mathrm{pmp}} T_{\mathrm{pmp}}\right) \\
& \quad \times \exp \left(-t^{2} / 2 T_{\mathrm{pmp}}^{2}\right) \cos \left(\omega_{\mathrm{pmp}} t\right),
\end{aligned}
$$

for a pulse of an oscillating electric field with amplitude $\boldsymbol{F}$, pulse width $T_{\mathrm{pmp}}$, and excitation energy $\omega_{\mathrm{pmp}}$.

\section{Results}

Because the energy of experimentally realized photoexcitation is generally higher than that of the conductivity peak, we set the photoexcitation energy $\omega_{\text {pmp }}$ to be $0.8 \mathrm{eV}$, which is higher than the energy of the calculated conductivity peak in both cases of charge-ordered (Fig. 1) and the Mott (Fig. 2) insulators.

For the charge-ordered insulator, Fig. 1a shows the time evolution of the hole density relative to the average at an originally hole-rich molecule $\mathrm{A}$ in Refs. [7, 8], $1.5-\left\langle n_{\mathrm{A}}(t)\right\rangle$, when the electron-phonon coupling is absent, $g=0$. The conductivity spectrum with polarization along the $a$-axis has a peak at $0.15 \mathrm{eV}$, which corresponds to the period of $42 \mathrm{eV}^{-1}=28 \mathrm{fs}$. After the photoexcitation, the corresponding oscillation of the hole density due to the charge transfer among neighboring molecules is conspicuous. It has a collective nature that comes from an oscillation between two charge configurations, which

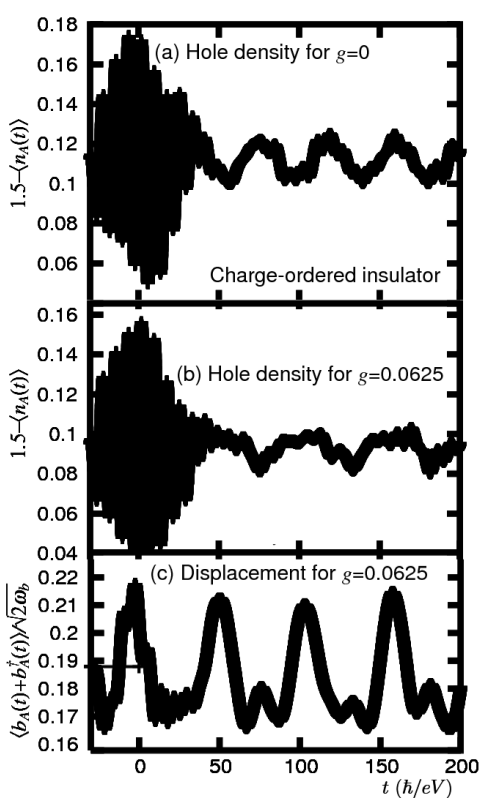

Fig. 1. Time dependences of (a) 1.5- $\left\langle n_{\mathrm{A}}(t)\right\rangle$ for $g=0$, (b) $1.5-\left\langle n_{\mathrm{A}}(t)\right\rangle$ for $g=0.0625$, and (c) $\left\langle b_{\mathrm{A}}(t)+\right.$ $\left.b_{\mathrm{A}}^{+}(t)\right\rangle / \sqrt{2 \omega_{b}}$ for $g=0.0625$ in the charge-ordered insulator during and after photoexcitation along the $a$-axis of $\omega_{\mathrm{pmp}}=0.8, T_{\mathrm{pmp}}=20$, and $F_{a}=1$.

are almost degenerate, i.e., the horizontal stripes on $\mathrm{A}$ and $\mathrm{B}$ molecules and those on $\mathrm{A}^{\prime}$ and $\mathrm{B}$ molecules in Refs. [7, 8]. Although the conductivity spectrum has individual modes above $0.285 \mathrm{eV}$, the corresponding oscillations below $22 \mathrm{eV}^{-1}$ are invisible. The collective charge-transfer excitation dominates the time evolution of the hole density even if its energy is far below the energy of photoexcitation.

Figure $1 \mathrm{~b}$ shows the time evolution of $1.5-\left\langle n_{\mathrm{A}}(t)\right\rangle$, when the electron-phonon coupling is turned on, $g=$ 0.0625. The conductivity spectrum with polarization along the $a$-axis has a peak at $0.115 \mathrm{eV}$, which corresponds to the period of $54 \mathrm{eV}^{-1}$. Its energy is softened by the electron-phonon coupling. The oscillation of the hole density due to the charge transfer among neighboring molecules is again conspicuous. Although the conductivity spectrum has individual modes above $0.235 \mathrm{eV}$, the corresponding oscillations below $27 \mathrm{eV}^{-1}$ are invisible. Figure 1c shows the time evolution of the molecular displacement at molecule $\mathrm{A},\left\langle b_{\mathrm{A}}(t)+b_{\mathrm{A}}^{+}(t)\right\rangle / \sqrt{2 \omega_{b}}$, when the electron-phonon coupling is turned on, $g=0.0625$. This quantity becomes zero all the time if $g=0$. The bare phonon energy of $0.2 \mathrm{eV}$ corresponds to the period of $31 \mathrm{eV}^{-1}$. In the time profile, the oscillation of the period of $54 \mathrm{eV}^{-1}$ is due to the coupling with the hole density at molecule $\mathrm{A}$. The time profile clearly shows interference between the charge transfer and this phonon mode.

For the Mott insulator, Fig. 2a shows the time evolution of the hole density relative to the average at molecule $1,1.5-\left\langle n_{1}(t)\right\rangle$, when the electron-phonon coupling is 


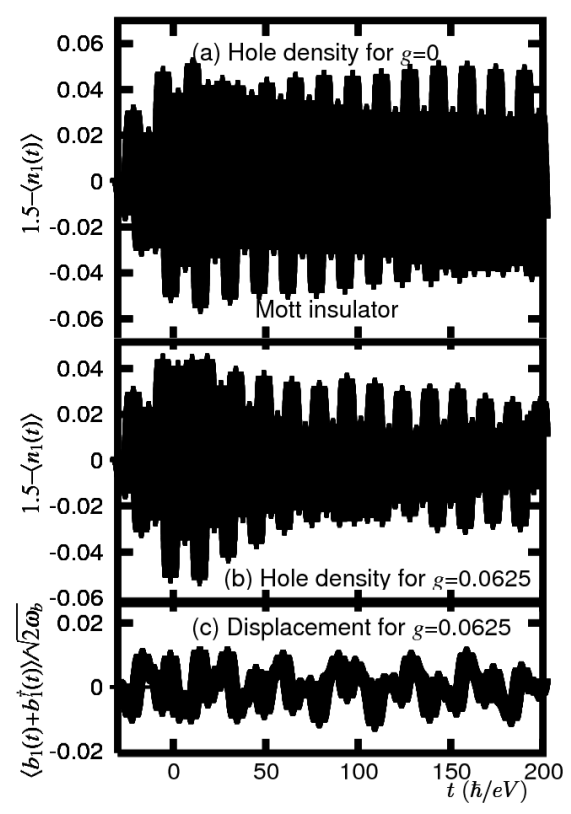

Fig. 2. Time dependences of (a) $1.5-\left\langle n_{1}(t)\right\rangle$ for $g=$ 0 , (b) $1.5-\left\langle n_{1}(t)\right\rangle$ for $g=0.0625$, and (c) $\left\langle b_{1}(t)+\right.$ $\left.b_{1}^{+}(t)\right\rangle / \sqrt{2 \omega_{b}}$ for $g=0.0625$ in the Mott insulator during and after photoexcitation along the $c$-axis of $\omega_{\text {pmp }}=0.8$, $T_{\mathrm{pmp}}=20$, and $F_{c}=1$.

absent, $g=0$. The conductivity spectrum with polarization along the $c$-axis has a peak at $0.415 \mathrm{eV}$, which corresponds to the period of $15 \mathrm{eV}^{-1}$. After the photoexcitation, the corresponding oscillation of the hole density due to the intradimer charge transfer is visible. It is an individual mode that comes from an oscillation between two molecules inside a dimer, where the coupling with neighboring dimers is weak. The energy of photoexcitation, $0.8 \mathrm{eV}$, corresponds to the period of $8 \mathrm{eV}^{-1}$. The corresponding oscillation is also present, though its amplitude is smaller than that of the intradimer charge-transfer excitation.

Figure $2 \mathrm{~b}$ shows the time evolution of $1.5-\left\langle n_{1}(t)\right\rangle$, when the electron-phonon coupling is turned on, $g=0.0625$. The conductivity spectrum with polarization along the $c$-axis has a peak at $0.43 \mathrm{eV}$, which corresponds to the period of $15 \mathrm{eV}^{-1}$. Its energy is almost unaffected by the electron-phonon coupling. The oscillation of the hole density due to the intradimer charge transfer is again visible. Figure 2c shows the time evolution of the displacement at molecule 1 , $\left\langle b_{1}(t)+b_{1}^{+}(t)\right\rangle / \sqrt{2 \omega_{b}}$, when the electron-phonon coupling is turned on, $g=0.0625$. The bare phonon energy of $0.2 \mathrm{eV}$ corresponds to the period of $31 \mathrm{eV}^{-1}$. The wiggling time profile is due to the coupling with the hole density at molecule 1 . Molecular displacements are forced to oscillate according to the electronic motion, but molecular vibrations are hardly excited (compare Figs. 1c and 2c).

\section{Conclusions}

Photoinduced charge and phonon dynamics in charge-ordered and Mott insulators are compared in two-dimensional three-quarter-filled extended HolsteinHubbard models on anisotropic triangular lattices for $\alpha$-(BEDT-TTF $)_{2} \mathrm{I}_{3}$ and $\kappa$-(BEDT-TTF $)_{2} \mathrm{X}$, respectively. Molecular vibrations are treated quantum-mechanically and the evolution of the exact many-electron-phonon wave functions is calculated on small clusters. For the charge-ordered insulator, we find a low-energy collective electronic motion originating from the oscillation between two nearly-degenerate charge configurations, even if the energy of photoexcitation is away from this energy. This motion strongly interferes with molecular vibrations. For Mott insulator, we find no such a collective electronic motion that interferes with or excites molecular vibrations. The electronic motion would be quickly dephased in realistic environments with thermal fluctuations. This explains why a collective electronic motion or quantum interference between electrons and phonons is not observed in the Mott-insulator phase.

\section{Acknowledgments}

This work was supported by Grants-in-Aid for Scientific Research (C) (grant No. 23540426), Scientific Research (B) (grant No. 20340101) and Scientific Research (A) (grant No. 23244062), and by "Grand Challenges in Next-Generation Integrated Nanoscience" from the Ministry of Education, Culture, Sports, Science and Technology of Japan.

\section{References}

[1] K. Yonemitsu, K. Nasu, Phys. Rep. 465, 1 (2008).

[2] N. Maeshima, K. Hino, K. Yonemitsu, Phys. Rev. B 82, 161105 (2010).

[3] Y. Kawakami, T. Fukatsu, Y. Sakurai, H. Unno, H. Itoh, S. Iwai, T. Sasaki, K. Yamamoto, K. Yakushi, K. Yonemitsu, Phys. Rev. Lett. 105, 246402 (2010).

[4] T. Takahashi, Y. Nogami, K. Yakushi, J. Phys. Soc. Jpn. 75, 051008 (2006).

[5] K. Kanoda, J. Phys. Soc. Jpn. 75, 051007 (2006).

[6] Y. Kawakami, S. Iwai, T. Fukatsu, M. Miura, N. Yoneyama, T. Sasaki, N. Kobayashi, Phys. Rev. Lett. 103, 066403 (2009).

[7] Y. Tanaka, K. Yonemitsu, J. Phys. Soc. Jpn. 79, 024712 (2010).

[8] S. Miyashita, Y. Tanaka, S. Iwai, K. Yonemitsu, J. Phys. Soc. Jpn. 79, 034708 (2010).

[9] K. Yonemitsu, S. Miyashita, N. Maeshima, J. Phys. Soc. Jpn. 80, 084710 (2011).

[10] T. Mori, H. Mori, S. Tanaka, Bull. Chem. Soc. Jpn. 72, 179 (1999). 